\title{
Optimization of Directional Antennas in Two-Dimensional Artificial Dielectrics
}

\author{
M. JanOWICZ ${ }^{a, *}$, M. RuseK ${ }^{b}$ AND A. ORŁOWSKI $I^{b}$ \\ ${ }^{a}$ Institut für Physik, Carl von Ossietzky Universität, 26111 Oldenburg, Germany \\ ${ }^{b}$ Instytut Fizyki, Polska Akademia Nauk \\ al. Lotników 32/46, 02-668 Warszawa, Poland
}

(Received November 5, 2003)

\begin{abstract}
We investigate numerically the problem of optimization of directional characteristics of dipole antennas located inside, or in the vicinity of, photonic crystals or more general artificial dielectrics, made of very thin perfectly conducting wires. We concentrate on two-dimensional propagation. Simulated annealing is used to find the distribution of wires which optimizes the directional pattern. It is demonstrated that high directivity can be obtained for systems containing a very small number of elements provided that the size and shape of the unit cell as well as the position of the radiating source with respect to the crystal are optimized. Building up of the radiation pattern is also illustrated with the help of the wave-optical rays.
\end{abstract}

PACS numbers: $41.20 . \mathrm{Jb}, 84.40 . \mathrm{Ba}, 42.25 . \mathrm{Bs}$

\section{Introduction}

The amount of applications of photonic crystals in various fields of optics, solid state physics, and technology has recently become rather impressive [1-3]. They include, among others, two-dimensional band-gap laser [4, 5], a new type of wave-guiding mechanism, associated with guiding and bending of electromagnetic waves through localized coupled-cavity modes [6], an increase in the photon lifetime along with extremely small group velocity at the coupled-cavity waveguide band edges [7]. In addition, there exist extremely interesting proposals for novel applications, like those concerning threshold-less semiconductor lasers $[1,8]$

* corresponding author, Alexander von Humboldt Fellow; permanent address: Instytut Fizyki, Polska Akademia Nauk, al. Lotników 32/46, 02-668 Warszawa, Poland 
and single-mode light-emitting diodes $[9,10]$. Important applications to the problem of design of microwave antennas [11] with a very high directivity have also been found [1, 12-16]. Both one-dimensional multilayer dielectric structures and three-dimensional photonic band-gap materials have been used. A particularly interesting configuration has been proposed in [16], where the photonic crystal with a negative effective refractive index has been applied to obtain an extremely high directivity. There can be no doubt that the novel optical materials will offer vast improvement in the antenna performance. The purpose of this paper is more modest, however. We would like to investigate to what extent one can improve the directivity of an antenna located inside or in the vicinity of an artificial dielectric (AD), either under the condition that our AD should in fact be a photonic crystal with a well-defined unit cell, or under no conditions at all, that is, admitting partially or fully disordered systems. It is shown that surprisingly few elements are necessary to obtain a very high directivity of antennas, if the positions of those elements are optimized. On the other hand, an unoptimized photonic crystal can work much poorer than an optimized random structure.

The main body of our work is organized as follows. In Sec. 2 we provide a detailed specification of a simple model of artificial dielectric which is used in the numerical simulations. Section 3 contains description and analysis of numerical results pertaining to the structure of optimized forms of ADs as well as the analysis of radiation patterns. In Sec. 4 we include some final remarks and discussion of the prospects of further research.

\section{Model and its solution}

Multi-parameter global optimization, which we attempt to perform here, is a very difficult task. In general, one cannot hope to obtain a really global minimum of a function of many variables; one can only hope to approach a relatively deep local minimum. And even if one is ready to accept this, there is an additional difficulty: global optimization is numerically expensive and time consuming. In order to minimize the time necessary for our numerical simulations, we decided — in this first attempt — to drastically reduce the complications and employ a very simplified model of elements which form the artificial dielectric. That is, our elements are assumed to be infinitely long parallel perfectly conducting wires. The source of radiation is another such wire antenna, parallel to all the other ones, with prescribed linear current density $I_{0}$. The point in the $(x, y)$ plane, where it is located, will be denoted by $\boldsymbol{r}_{0}$. This model allows us to perform a large part of calculations analytically, and they are in addition rather simple.

Since all our sources are parallel infinite wires, the propagation of radiation is just two-dimensional, so that we consider here only the propagation of $E$-waves. The time dependence is assumed to have the form $\exp (-\mathrm{i} \omega t)$. Let us choose the $z$-axis to be parallel to the wires. Then the Maxwell equations for the components $E_{z}$ of the electric field and $H_{x}, H_{y}$ of the magnetic field take the following form: 


$$
\begin{aligned}
& \frac{\partial E_{z}}{\partial y}=\mathrm{i} \mu_{0} \omega H_{x}, \\
& \frac{\partial E_{z}}{\partial x}=-\mathrm{i} \mu_{0} \omega H_{y}, \\
& \frac{\partial H_{y}}{\partial x}-\frac{\partial H_{x}}{\partial y}=-\mathrm{i} \epsilon_{0} \omega E_{z}+J_{z},
\end{aligned}
$$

where $\mu_{0}$ is the vacuum magnetic permeability, $\epsilon_{0}$ is the vacuum electric permittivity, and $J_{z}$ is the current density including currents induced in the wires.

From the above equations we immediately obtain the Helmholtz equation for $E_{z}$

$$
\left(\nabla_{t}^{2}+k^{2}\right) E_{z}=-i \mu_{0} \omega J_{z}
$$

where $\nabla_{t}^{2}=\partial^{2} / \partial x^{2}+\partial^{2} / \partial y^{2}$. The solution to the problem (2) is given by

$$
E_{z}(\boldsymbol{r})=\mathrm{i} \mu_{0} \omega \lim _{\delta \rightarrow 0} \int_{S-S_{\delta}} G\left(\boldsymbol{r}-\boldsymbol{r}^{\prime}\right) J_{z}\left(\boldsymbol{r}^{\prime}\right)+E_{z}^{0}(\boldsymbol{r})+\frac{1}{\mathrm{i} \omega \epsilon_{0}} \hat{L} J_{z}(\boldsymbol{r}) .
$$

In the above equation $S$ is the total two-dimensional space, whereas $S_{\delta}$ is a sum of "principal surfaces" which exclude singularities of $G\left(\boldsymbol{r}-\boldsymbol{r}^{\prime}\right) ; S_{\delta}$ becomes infinitesimally small as their maximum chord length approaches zero [17]. In our case, the surface $S_{\delta}$ comprises $n$ small surfaces covering the points in the $(x, y)$ plane, where the wires are located. The field $E_{z}^{0}$ is the solution to a homogeneous Helmholtz equation, which is in our case the field due to the prescribed antenna current. Finally, the source dyadic $L$ appears here to make the solution rigorous in the region, where sources are present. Indeed, as has been shown by Yaghjian in Ref. [17], the dyadic $\hat{L}$ is, in generic case, necessary to obtain correct solutions in that region. However, the appropriate $L \mathrm{~s}$ in two-dimensional problems are transverse, that is, they do not contain any $m z$ component $(m=x, y, z)$. This means that in our case the last term in Eq. (3) actually vanishes, and the resulting electric field simplifies to

$$
E_{z}(\boldsymbol{r})=\mathrm{i} \mu_{0} \omega \sum_{j=1}^{n} G\left(\boldsymbol{r}-\boldsymbol{r}_{j}\right) I_{j}+E_{z}^{0}(\boldsymbol{r}),
$$

because all wires are infinitely thin, so that

$$
J_{z}(\boldsymbol{r})=\sum_{j=1}^{n} I_{j} \delta\left(\boldsymbol{r}-\boldsymbol{r}_{j}\right),
$$

where $I_{j}, j=1,2, \ldots, n$ are induced currents associated with each wire. We must remember, however, that - when calculating the field at each point, where the current is different from zero - an infinitesimally small surface surrounding the point with non-zero current should be excluded from integration. In practice, this leads to the vanishing of diagonal elements of a characteristic matrix $g_{i j}$ (see below), which is to be inverted to obtain currents $I_{j}$. 
Since our wires are infinitely conducting, the electric field must vanish at each point $r_{i}$, so that

$$
0=E_{z}\left(r_{i}\right)=\mathrm{i} \mu_{0} \omega \sum_{j=1}^{n} G\left(r_{i}-r_{j}\right) I_{j}+E_{z}^{0}\left(r_{i}\right) .
$$

The Green function of the Helmholtz equation which satisfies the equation

$$
\left(\nabla_{t}^{2}+k^{2}\right) G\left(r, r^{\prime}\right)=-\delta\left(r-r^{\prime}\right)
$$

is given by [18]

$$
G\left(r-r^{\prime}\right)=\frac{1}{4} \mathrm{i} H_{0}^{1}\left(k\left|r-r^{\prime}\right|\right)
$$

where $H_{m}^{1}(x)$ is the $m$ th-order Hankel function of the first kind, and argument $x$ [19]. Thus, we can rewrite Eq. (5) as a system of linear equations to obtain the current densities $I_{j}$

$$
\sum_{j=1}^{n} g_{i j} I_{j}=e_{0 i}
$$

where

$$
g_{i j}=\frac{1}{4} \mathrm{i} H_{0}^{1}\left(k\left|r_{i}-r_{j}\right|\right)
$$

and $g_{i i}=0$, while

$$
e_{0 i}=-\frac{1}{4} \mathrm{i} H_{0}^{1}\left(k\left|\boldsymbol{r}_{i}-\boldsymbol{r}_{0}\right|\right)
$$

From the Maxwell equations we obtain the complete solution for the fields

$$
\begin{aligned}
& E_{z}(\boldsymbol{r})=-\frac{1}{4} \mu_{0} \omega \sum_{j=0}^{n} H_{0}^{1}\left(k\left|\boldsymbol{r}-\boldsymbol{r}_{j}\right|\right) I_{j}, \\
& H_{x}(\boldsymbol{r})=-\frac{1}{4} \mathrm{i} k \sum_{j=0}^{n} \frac{y-y_{j}}{\left|\boldsymbol{r}-r_{j}\right|} H_{1}^{1}\left(k\left|\boldsymbol{r}-\boldsymbol{r}_{j}\right|\right) I_{j}, \\
& H_{y}(\boldsymbol{r})=\frac{1}{4} \mathrm{i} k \sum_{j=0}^{n} \frac{x-x_{j}}{\left|\boldsymbol{r}-\boldsymbol{r}_{j}\right|} H_{1}^{1}\left(k\left|\boldsymbol{r}-\boldsymbol{r}_{j}\right|\right) I_{j} .
\end{aligned}
$$

Using asymptotic expansions of the Hankel functions, we obtain the following formulae for the Cartesian components of the Poynting vector in the radiation zone:

$$
\begin{aligned}
& S_{x}=-\frac{1}{2} E_{z} H_{y}^{\star}=\frac{\mu_{0} \omega}{16 \pi r} \cos (\phi) \sum_{j, l} \exp \left[\mathrm{i} k\left(\left|r-r_{j}\right|-\left|r-r_{l}\right|\right)\right] I_{j} I_{l}^{*}, \\
& S_{y}=\frac{1}{2} E_{z} H_{x}^{\star}=\frac{\mu_{0} \omega}{16 \pi r} \sin (\phi) \sum_{j, l} \exp \left[\mathrm{i} k\left(\left|r-r_{j}\right|-\left|r-r_{l}\right|\right)\right] I_{j} I_{l}^{*},
\end{aligned}
$$


where $(r, \phi)$ are the polar components of the observation point $r$. Since $r \gg r_{j}$ for every $j$, the argument of the exponential function can be further simplified to give

$$
\left|r-r_{j}\right|-\left|r-r_{l}\right|=r_{l} \cos \left(\phi-\phi_{l}\right)-r_{j} \cos \left(\phi-\phi_{j}\right)
$$

The (unnormalized) far-zone radiation pattern becomes thus:

$$
\begin{aligned}
& P(\phi)=r S_{r}=r\left(S_{x} \cos \phi+S_{y} \sin \phi\right)= \\
& \quad \frac{\mu_{0} \omega}{16 \pi} \sum_{j, l} \exp \left[\mathrm{i} k\left(r_{l} \cos \left(\phi-\phi_{l}\right)-r_{j} \cos \left(\phi-\phi_{j}\right)\right)\right] I_{j} I_{l}^{\star}
\end{aligned}
$$

and is explicitly real.

\section{Numerical results}

We have decided to define the function to be optimized as follows. We have taken a large natural number $N$ (eventually $N$ was chosen to be equal to 1440 ), and, for $\phi_{m}=-\pi+2 \pi m / N, m=0,1,2, \ldots N-1$, have defined two sums

$$
U_{1}=\sum_{-\phi_{0} \leq \phi_{m} \leq \phi_{0}} P\left(\phi_{m}\right)
$$

and

$$
U_{2}=\sum_{m=0}^{N-1} P\left(\phi_{m}\right)
$$

for a small angle $\phi_{0}$. The optimization problem has been defined as that of minimization of the following ratio:

$$
t\left(r_{j} ; \phi_{0}\right)=\frac{U_{2}}{U_{1}}
$$

We have experimented with several values of the angle $\phi_{0}$ before choosing it to be equal to $\pi / 72$.

Our optimizations have been performed using two freely available Fortran routines: the simulated annealing code simann, available from www.netlib.org, and the program DIRect by J. Gablonsky. The frequency of radiation has been chosen to be equal to $10 \mathrm{GHz}$, but all other frequencies are obviously available by trivial rescaling. In our tables and figures we have used SI units to specify the (sub)optimal location of the wires. As already stated above, the absolute optimum is extremely difficult to obtain, and we can only claim that we have reached a deep local minimum, or have approached it closely.

Optimization of antennas in the vicinity of potentially completely disordered system formed the first part of our numerical experiments. The optimization routines have been allowed to change both coordinates of all (4 or 8) wires - they have only been constrained by the requirement that the whole structure is invariant under reflections with respect to $x$ axis. In this sense we call such structures 
"random". The local minimum found gives a rather poor value of $t$; still, the maximum near the angle 0 is well visible, and turns out to be much better than for an unoptimized crystalline structure. The coordinates of wires corresponding to the local minimum are given in Table I and illustrated in Fig. 1, while the normalized radiation patterns are shown in Fig. 2.

TABLE I

Coordinates of wires are shown which resulted from an attempt to optimize the radiation pattern without imposing the condition of regularity of the structure to be formed by the wires, except of the reflection symmetry with respect to the $y$ axis.

\begin{tabular}{c|c|c|c|c|c|c|c|c}
\hline \hline \multicolumn{7}{c}{ A. Structure with four wires } \\
\hline$x(m)$ & 5.8310 & -0.0385 & 5.8310 & -0.0385 & & & & \\
\hline$y(m)$ & -0.2395 & 0.1395 & 0.2395 & -0.1395 & & & & \\
\hline \multicolumn{7}{c}{ B. Structure with eight wires } \\
\hline$x(m)$ & 8.9865 & 3.3558 & -4.6385 & 4.6585 & 8.9865 & 3.3558 & -4.6385 & 4.6585 \\
\hline$y(m)$ & -0.0579 & -5.6703 & -0.6745 & -0.4340 & 0.0579 & 5.6703 & 0.6745 & 0.4340
\end{tabular}

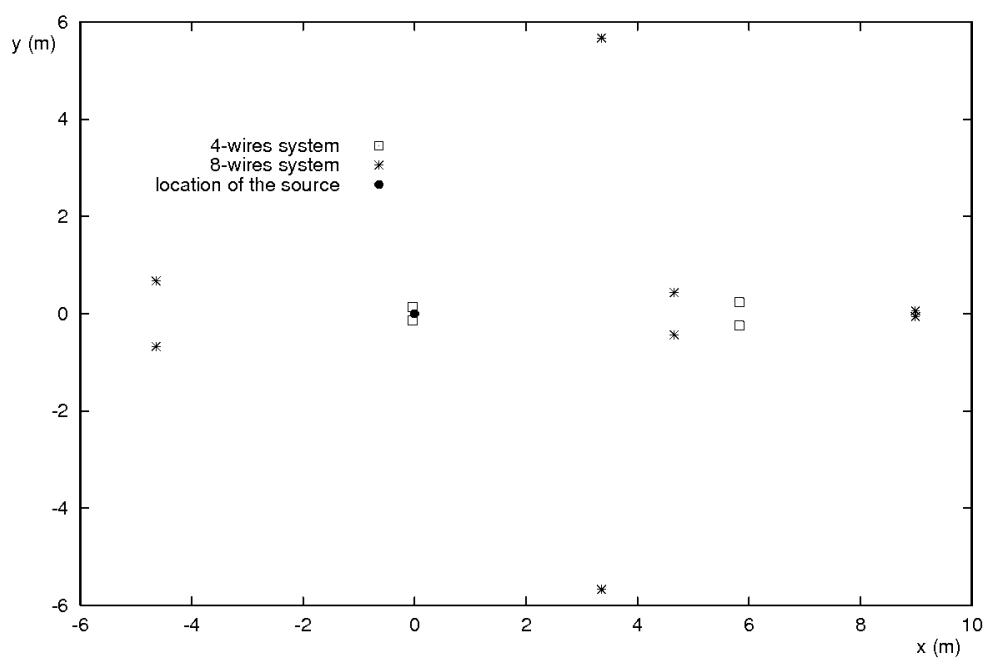

Fig. 1. Suboptimal locations of wires in structures for which optimization involved changing both coordinates $x$ and $y$. Systems with 4 and 8 wires are shown. The data have been taken from Table I. The wavelength is equal to $0.189 \mathrm{~m}$.

"Normalized radiation pattern" means that we actually plot the following quantity:

$$
P_{1}(\phi)=P(\phi) / \sum_{m=0}^{N-1} P\left(\phi_{m}\right) .
$$




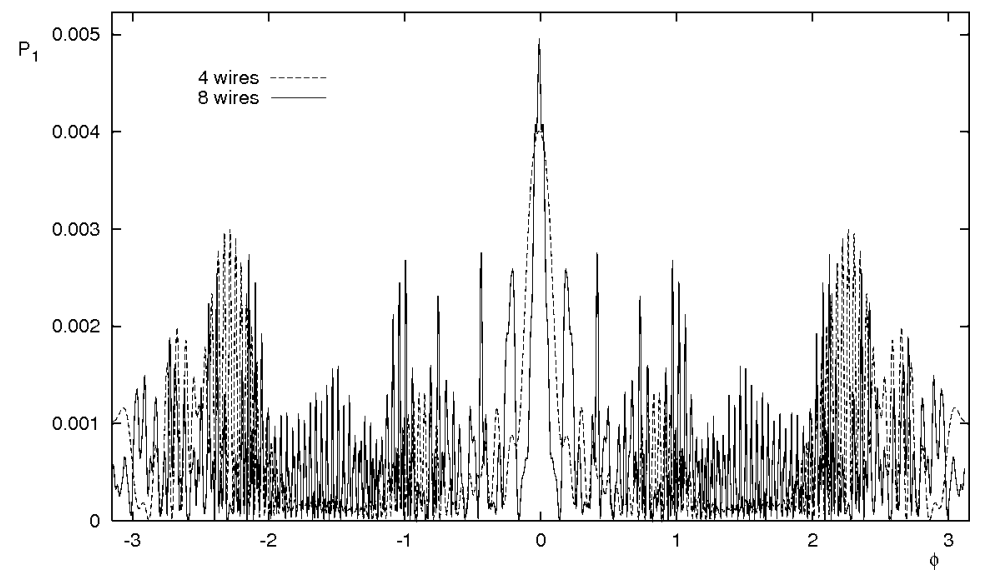

Fig. 2. Normalized radiation pattern for the systems of wires located according to Table I and Fig. 1.

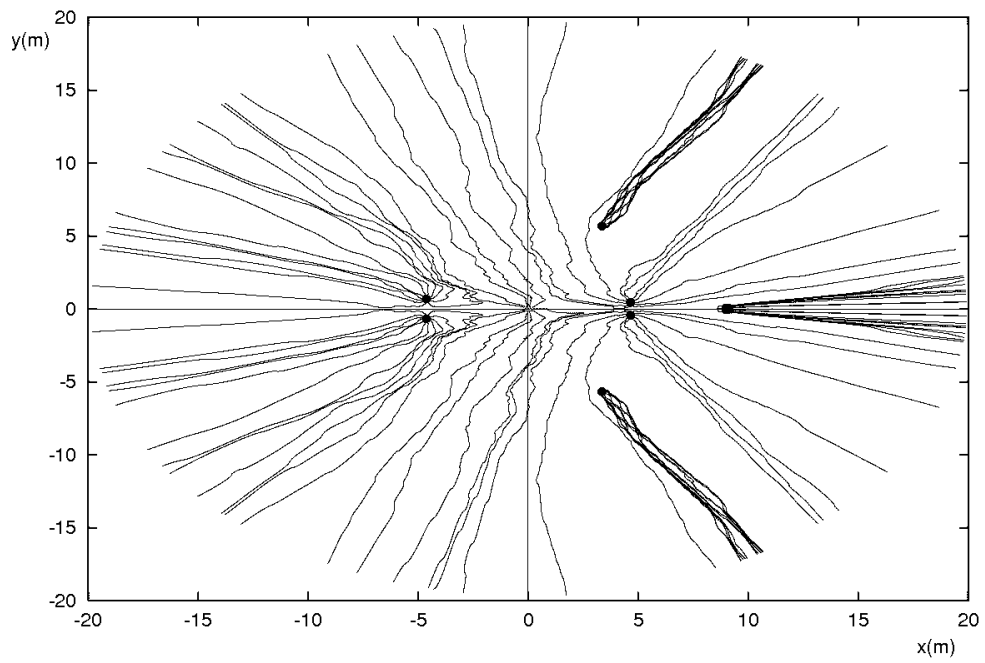

Fig. 3. Wave-optical rays for the 8-wires two-dimensional "random" structure.

In Fig. 3 we have plotted 99 trajectories of the wave-optical rays, that is, the curves which are tangent to the Poynting vector at each point. As they are obtained by integrating numerically the following equation:

$$
\frac{\mathrm{d} \boldsymbol{r}}{\mathrm{d} \tau}=\boldsymbol{S}(\boldsymbol{r})
$$

(where $\tau$ is an arbitrary parameter), they cannot intersect each other, unlike the geometrooptical rays. This follows from the Picard theorem about the local uniqueness of the dependence of solutions of ordinary differential systems - with Lipschitzian right-hand side - on initial conditions. However, the wave-optical rays 
can run quite erratically, as is seen in Fig. 3. This figure also well illustrates how the far-zone radiation pattern for angles close to zero is built (let us notice that we have used Eqs. (11) to compute the right-hand side of (18), without using asymptotic approximations for the Hankel functions). An interesting effect is visible, which will be even more pronounced in our further examples: although the far-zone "main" maximum (i.e., that for small angles) appears, in the wave-optical-ray picture, already for the distances from the system which are comparable with the distances between the wires, the other maxima require considerable redistribution of rays on the still much larger scale.

TABLE II

Coordinates of wires are shown which resulted from optimization under the condition that all the wires are located on the axis of abscissas.

\begin{tabular}{l|l|l|l|l|l|l|l|l}
\hline \hline \multicolumn{7}{c}{ A. Structure with four wires } \\
\hline$x(m)$ & -19.3643 & -9.3807 & -1.5126 & -0.3275 & & & & \\
\hline \multicolumn{7}{c}{ B. Structure with eight wires } \\
\hline$x(m)$ & -23.1057 & -11.8038 & -3.9357 & -1.0489 & -0.1379 & -0.0784 & -0.02399 & -0.0100
\end{tabular}

Our next optimization involved an elementary partially disordered system. That is, all the wires have been constrained to lie on the $x$ axis, but their $x$-coordinates have not been arranged in the crystal-like structure, but rather allowed to "move" freely to look for the minimum of $t$. Our Table II and Fig. 4 show the suboptimal $x$-coordinates of the wires for $n$ equal to 4 and 8 .

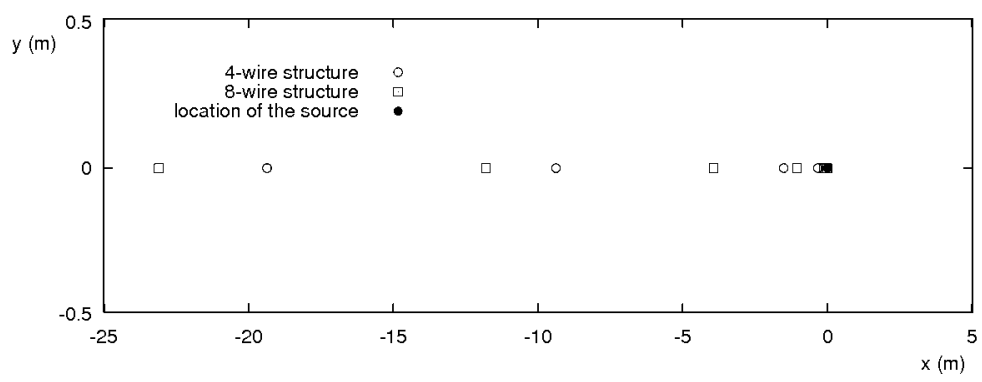

Fig. 4. Suboptimal locations of wires in structures for which optimization involved changing only $x$ coordinates of the wires while their $y$ coordinates have been equal to zero. Systems with 4 and 8 wires are shown. The data have been taken from Table II.

We can see that the found local minimum corresponds to a rather disordered system. Nevertheless, the directivity, shown in Fig. 5 turns out to be quite considerable, although much worse than that in the case of the crystalline AD of the next example. 


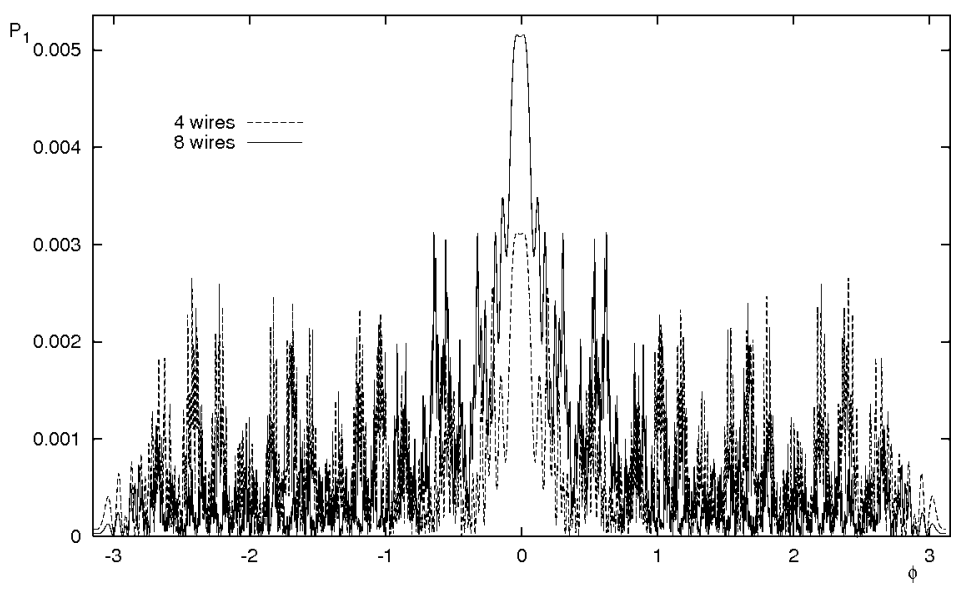

Fig. 5. Normalized radiation pattern for the system of 4 and 8 wires located according to Table II and Fig. 4.

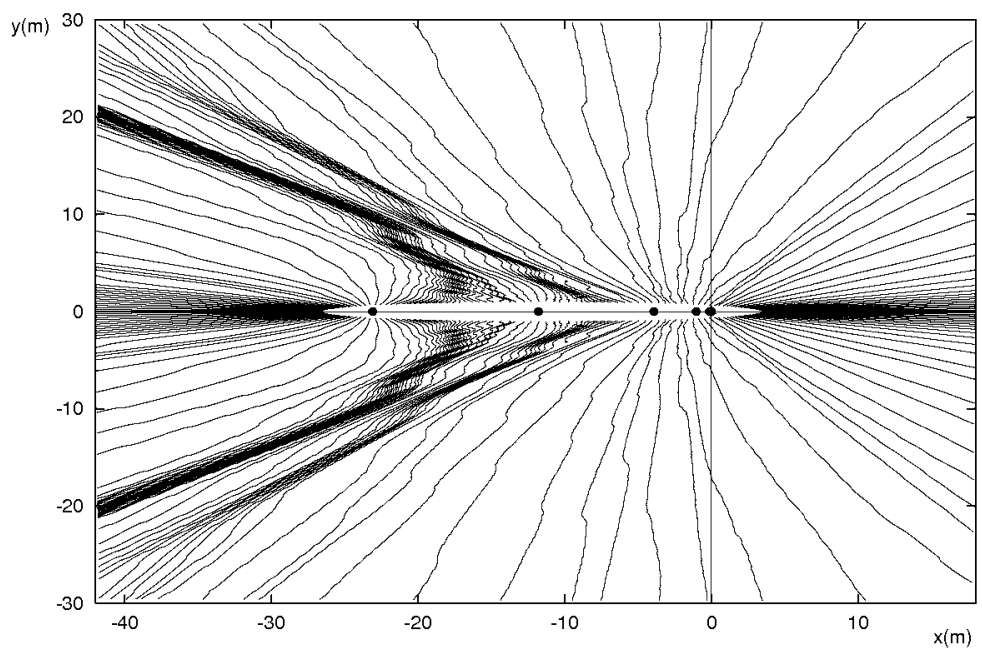

Fig. 6. Wave-optical rays for the 8-wires one-dimensional "random" structure.

In Fig. 6 we have plotted corresponding wave-optical rays, but - unlike in Fig. 3 - this time our rays start from a long and thin ellipse surrounding the wires. Building-up of the diffraction maximum near $\phi=0$ is again excellently visible, but considerable rearrangements must evidently happen in far zone since there are no far-zone maxima near the angle $\phi=\pi$.

Furthermore, we have assumed that the conducting wires form a photonic crystal with the length and width of the unit cell given by $2 a$ and $2 b$, and the shift of the "center of mass" of the crystal with respect to the source denoted by $d$. 
TABLE III

Coordinates of wires are shown which resulted from optimization under the condition that the wires form a small crystalline structure with the unit cell length $2 a$, width $2 b$, and the "center of mass" of the crystal located at $d$.

\begin{tabular}{l|c|c|c}
\hline \hline \multicolumn{4}{|c}{ Parameters of crystal structure } \\
\hline & 4 wires & 16 wires & 36 wires \\
\hline$a(m)$ & 2.7377 & 4.1648 & 4.1665 \\
\hline$b(m)$ & 0.0503 & 0.1524 & 0.0567 \\
\hline$d(m)$ & 2.8815 & 15.9225 & 16.0523
\end{tabular}

The optimized parameters $a, b, d$ are given in Table III for three numbers of wires to form the crystal - they were equal to 4,16 , and 36 . Figure 7 illustrates the location of wires according to Table III.

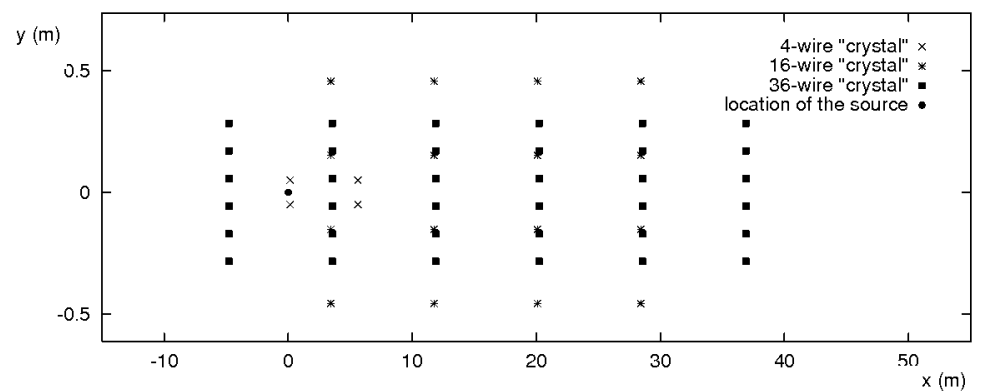

Fig. 7. Suboptimal locations of wires in rectangular "crystalline" structures for which optimization involved changing the size of elementary cell as well as the overall location of wires with respect to the source of radiation. Systems with 4, 16, and 36 wires are shown. The data have been taken from Table III.

Figures 8,9 , and 10 provide corresponding radiation patterns. The fact that the directivity improves when the number of elements becomes larger seems rather intuitive. In particular, the directivity for the case $n=36$ seems to be quite satisfactory, although we have found it difficult to remove the annoying local minimum at $\phi=0$. In Fig. 11 we provide the corresponding trajectories of wave-optical rays. Let us notice that the obvious maximum at $\phi=\pi$, which disappears for still larger distances, is not an artifact connected with a peculiar choice of the initial conditions for the trajectories, which, as in Fig. 6, start from a highly eccentric ellipse surrounding the wires.

The following observation is perhaps of some interest. The simulated annealing routine simann requires some initial guess as an input. Normally, this initial 


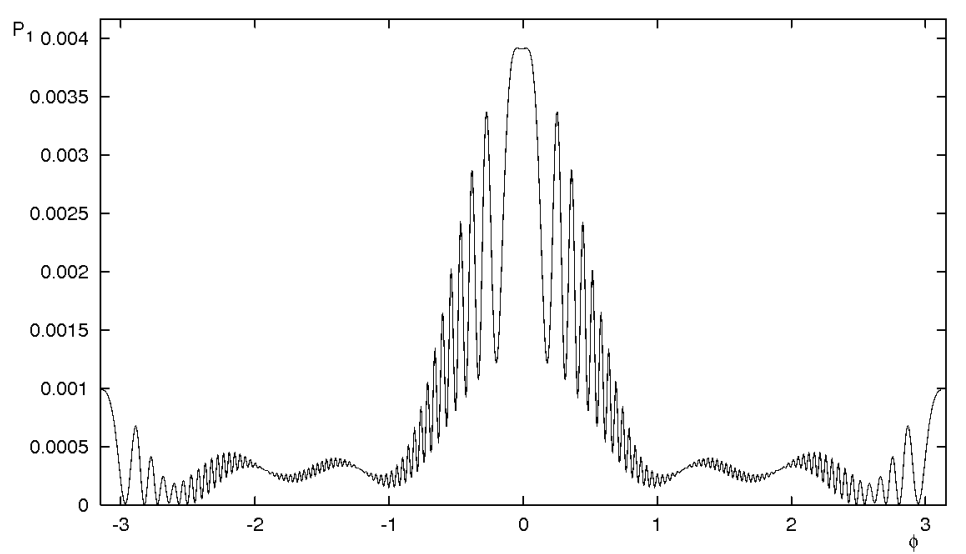

Fig. 8. Normalized radiation pattern for the systems of 4 located according to Table III and Fig. 7.

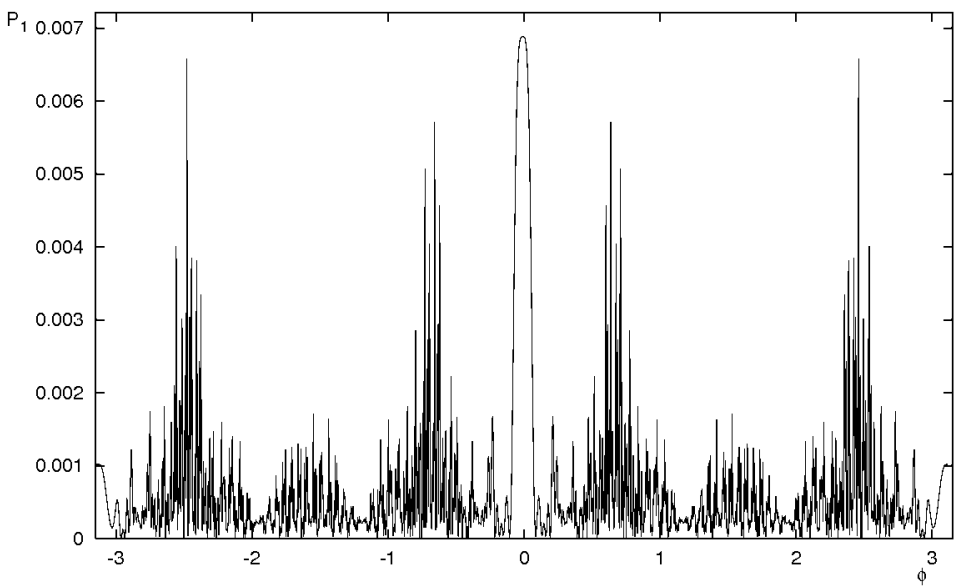

Fig. 9. Normalized radiation pattern for the systems of 16 located according to Table III and Fig. 7.

guess have been made using the random number generator ranlux. However, if the initial guess contained the positions of wires corresponding to a previously optimized photonic crystal with the same number of elements, simann has still been able to find a little bit "better" positions of wires, with slightly improved directivity, but with the crystalline structure somewhat "spoiled". But the defects have been extremely small, the lengths and widths of deformed unit cells have deviated by no more than $0.01 \%$, and the improvement in our $t$ function has been very insignificant. Therefore, although the strategy itself, i.e., first optimize the location of elements in a crystalline structure, then feed the optimization routine with their positions and try to find a better one, seems to be advisable, in this case we have not obtained any substantial changes. 


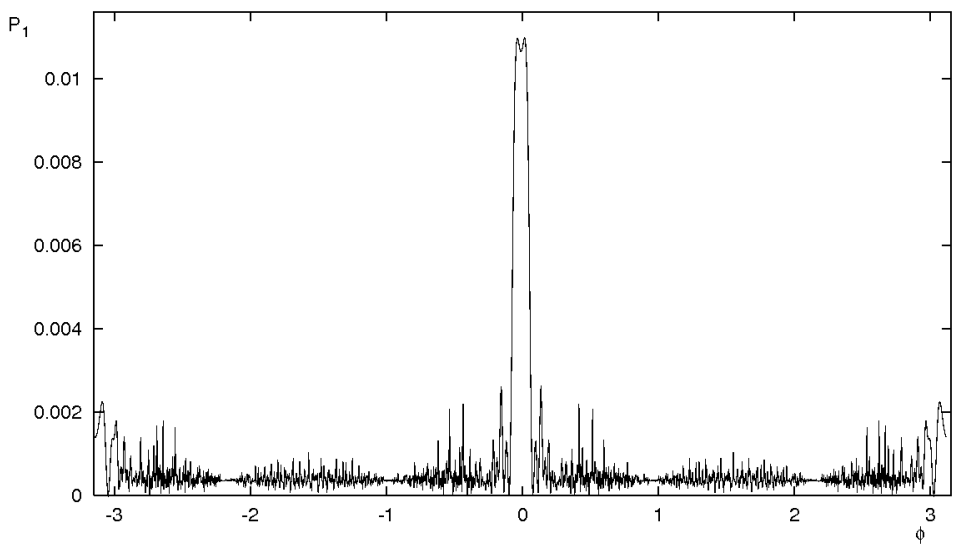

Fig. 10. Normalized radiation pattern for the systems of 36 located according to Table III and Fig. 7.

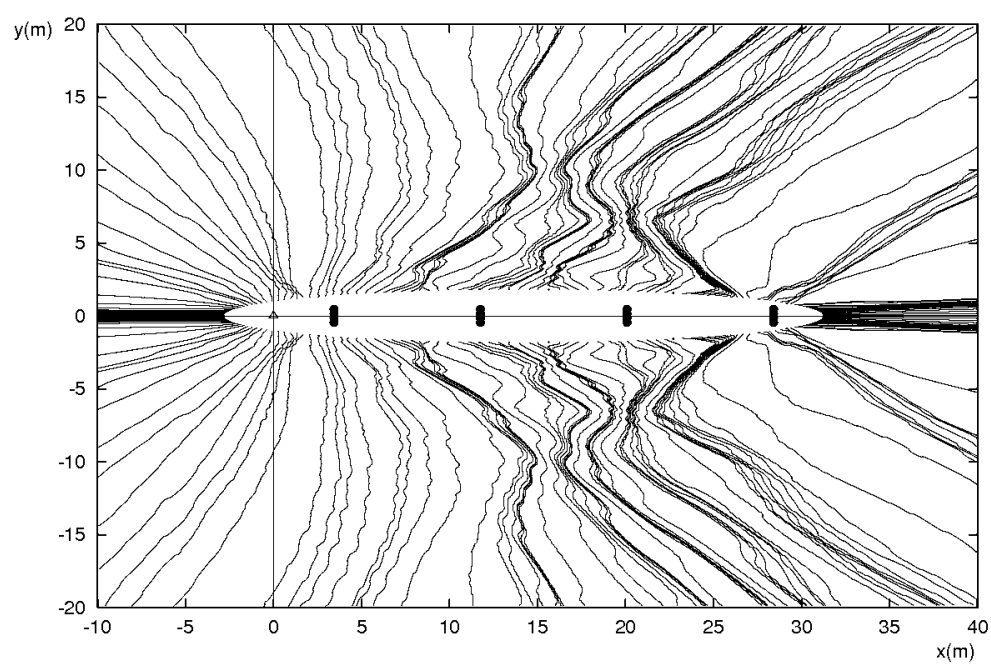

Fig. 11. Wave-optical rays for the 16-wires "crystalline" structure.

The form of our suboptimal small crystalline structures has suggested that a waveguide-like structure, empty inside, and located close to the radiation source, can also provide a useful system to obtain a high directivity. In our final numerical experiments, we have tried to optimize the "width" of the waveguide, the distance between elements, and the overall location of the waveguide with respect to the source. Suboptimal location of the elements, for 17 and 31 wires, is shown in Fig. 12.

In Figs. 13 and 14 we have plotted the radiation patterns for the waveguide-like structures with 17 and 31 wires. It is seen that the directivity is at least comparable with that of the crystal-like systems, but, not surprisingly, we have 


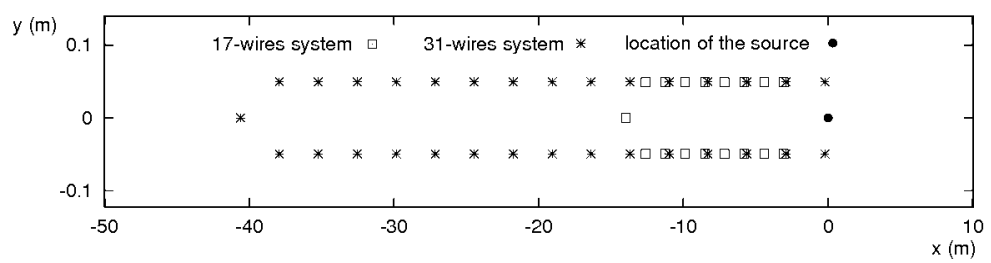

Fig. 12. Suboptimal locations of wires in "waveguide" structures for which optimization involved changing the width of the waveguide, the distance between the elements forming its "surface" as well as the overall location of wires with respect to the source of radiation. Systems with 17 , and 31 wires are shown.

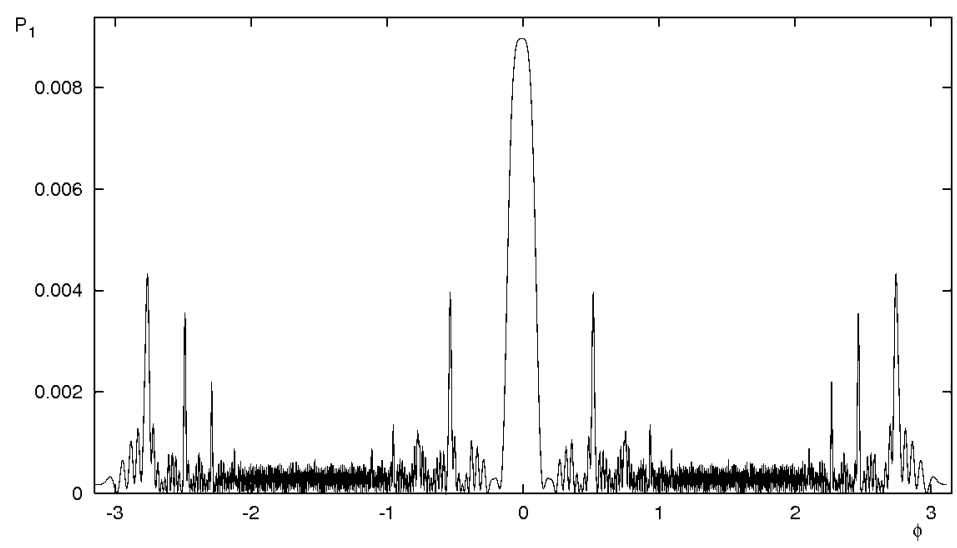

Fig. 13. Normalized radiation pattern for the systems of 17 located according to Fig. 12.

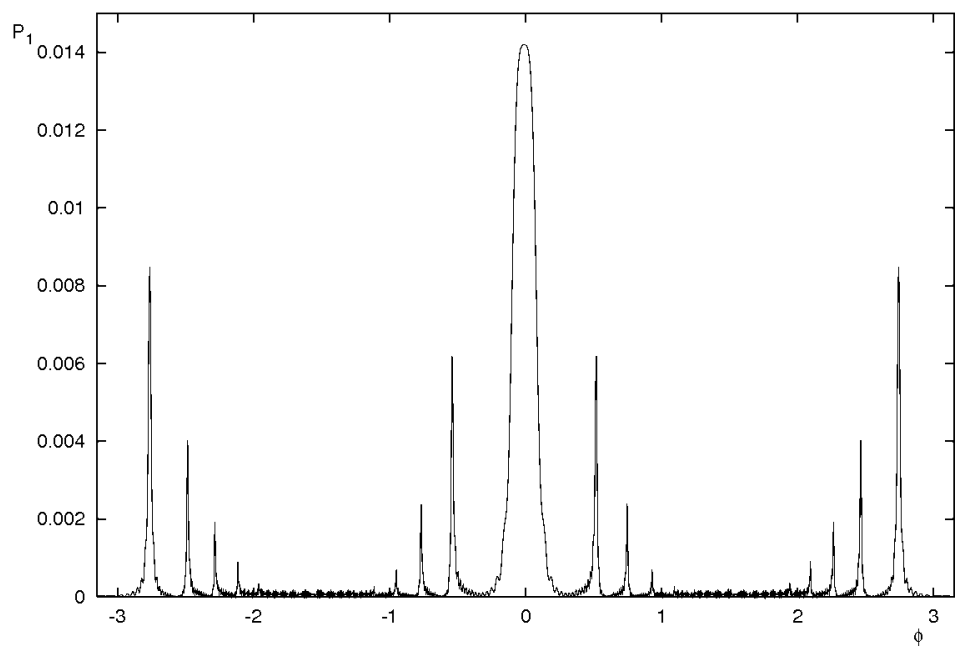

Fig. 14. Normalized radiation pattern for the systems of 31 located according to Fig. 12. 
encountered serious difficulties to eliminate narrow side diffraction maxima. These could not be eliminated even though we have added a penalty function to our function $t$ in order to minimize also the ratio of heights of side maxima to the height of the main maximum.

For the system comprising 17 wires we also provide the illustration of how the far-zone radiation pattern emerges from the wave-optical rays, see Fig. 15.

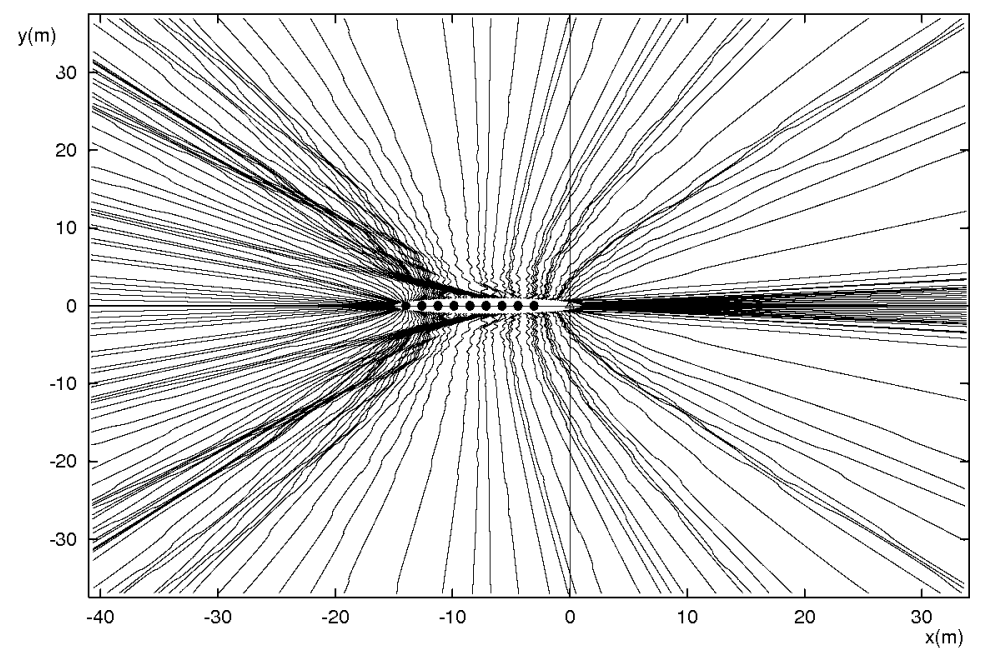

Fig. 15. Wave-optical rays for the 17 -wires "waveguide" structure.

It is to be noticed that it would be very interesting to investigate the fine structure of radiation inside photonic crystals and waveguide-like systems in the spirit of [20]. The wave-optical rays (together with the lines of constant amplitude and of constant phase) are an ideal tool for such an analysis. In particular, the distribution of stationary points and the structure of separatrices bring about valuable information on the electromagnetic fields. In this paper we have not touched these fascinating topics.

\section{Final remarks}

In this work we have analyzed the possibilities of optimization of radiation patterns of idealized antennas emitting only $E$ waves, located inside, or in the vicinity of, artificial dielectrics. Artificial dielectrics considered here have been assumed to comprise perfectly conducting infinitely thin parallel wires, so that the propagation problem has been restricted to two dimensions. We have shown that very high directivity can be achieved for (sub-)optimal positions of conducting elements provided that they form a photonic crystal or a waveguide-like system, even if the number of those elements is rather small, of the order of 10 . The 
half-power beam-width has been as small as $8^{\circ}$ for just about 30 wires forming a crystal. In addition, we have demonstrated that it is also possible to obtain a quite considerable directivity from partially disordered, or even completely disordered ADs, although their directional characteristics have been much worse than those of optimized photonic crystals.

There are some desirable features absent in this research, which we plan to include in our future work. Firstly, we are going to get rid of the assumption that the elements are parallel infinitely long wires, and consider full three-dimensional propagation. Only then our optimization can be compared with the results of [13-16]. Secondly, the assumption that the elements to form ADs are perfectly conducting must be relaxed. Thirdly, we have not considered here the problem of efficiency of antennas. If numerical optimization like that performed here has to have real applications, the gain must be optimized together with the directivity. In addition, it is also possible to state the optimization problems as follows: try to find the minimum number of elements and optimal parameters of the crystal, such that the desired values of directivity and the gain can be achieved. This is a mixed-integer optimization problem, the numerical solution of which is highly non-trivial, but conceivable.

\section{Acknowledgment}

This work has been supported by the State Committee for Scientific Research (Poland) grant No. 2 P03B 04419.

\section{References}

[1] Photonic Crystals and Light Localization in the 21st Century, Ed. C.M. Soukoulis, Kluwer, Dordrecht 2001.

[2] J.D. Joannopoulos, R.D. Meade, J.N. Winn, Photonic Crystals: Molding the Flow of Light, Princeton University Press, Princeton 1995.

[3] Photonic Band Gap Materials, Ed. C.M. Soukoulis, Kluwer, Dordrecht 1996.

[4] O. Painter, R.K. Lee, A. Scherer, A. Yariv, J.D. O'Brien, P.D. Dapkus, I. Kim, Science 284, 5421 (1999).

[5] A. Mekis, M. Meier, A. Dodabalapur, R.E. Slusher, J.D. Joannopoulos, Appl. Phys. A 69, 111 (1999).

[6] M. Bayindir, B. Temelkuran, E. Ozbay, Phys. Rev. B 61, R11855 (2000).

[7] M. Bayindir, E. Ozbay, Phys. Rev. B 62, R2247 (2000).

[8] P.R. Villeneuve, S. Fan, J.D. Joannopoulos, K.Y. Lim, G.S. Petrich, L.A. Kołodziejski, R. Reif, Appl. Phys. Lett. 67, 167 (1995).

[9] P.L. Gourley, J.R. Wendt, G.A. Vawter, T.M. Brennan, B.E. Hammons, Appl. Phys. Lett. 64, 687 (1994).

[10] J.P. Dowling, M. Scalora, M.J. Bloemer, C.M. Bowden, J. Appl. Phys. 75, 1896 (1994) 
[11] C.A. Balanis, Antenna Theory. Analysis and Design, Wiley, New York 1982.

[12] M. Thevenot, C. Cheype, A. Reneix, B. Jecko, IEEE Trans. Antennas and Propagation 47, 2115 (1999).

[13] M.M. Sigalas, R. Biswas, Q. Li, D. Crouch, W. Leung, R. Jacobs-Woodbury, B. Lough, S. Nielsen, S. McCalmont, G. Tuttle, K.-M. Ho, Microwave and Opt. Tech. Lett. 15, 153 (1997).

[14] B. Temelkuran, M. Bayindir, E. Ozbay, R. Biswas, M. Sigalas, G. Tuttle, K.-M. Ho, J. Appl. Phys. 87, 603 (2000).

[15] R. Biswas, E. Ozbay, B. Temelkuran, M. Bayindir, M.M. Sigalas, K.-M. Ho, in Ref. [1], p. 321.

[16] S. Enoch, G. Tayeb, P. Sabouroux, N. Guerin, P. Vincent, Phys. Rev. Lett. 89, $213902(2002)$.

[17] A.D. Yaghjian, Proc. IEEE 68, 248 (1980).

[18] L.B. Felsen, N. Marcuvitz, Radiation and Scattering of Waves, IEEE Press and Oxford University Press, New York 1994.

[19] M. Abramowitz, I. Stegun, Handbook of Mathematical Functions, Dover, New York 1980.

[20] W. Żakowicz, Phys. Rev. E 64, 066610 (2001). 